Supporting information for publishing

\title{
Achieving High Thermoelectric Performance of Tetrahedrites by Adjusting the Electronic Density of State and Enhancing Phonon \\ Scattering
}

Lulu Huang,a,b, Yuan Kong",c , Jian Zhanga ${ }^{a^{*}}$, Rui Xu' ${ }^{\text {d }}$ Chen Zhu ${ }^{\text {a,b }}$, Jie Wu ${ }^{\text {a,b }}$, Jabbar Bushra $^{\mathrm{a}, \mathrm{b}}$, Di Li ${ }^{\mathrm{a}^{*}}$, Zhaoming Wang ${ }^{\mathrm{a}}$, Xiaoying Qin ${ }^{\mathrm{a}^{*}}$

a Key Laboratory of Materials Physics, Institute of Solid State Physics, Chinese Academy of Sciences, 230031 Hefei, PR China

b University of Science and Technology of China, Hefei 230026, China

c Hefei National Laboratory for Physical Sciences at the Microscale and Department of Chemical Physics and Synergetic Innovation Center of Quantum Information and Quantum Physics, University of Science and Technology of China, Hefei, Anhui 230026, China

d MIIT Key Laboratory of Advanced Metallic and Intermetallic Materials Technology, School of Materials Science and Engineering, Nanjing University of Science and Technology, Nanjing 210094, China.

\# These authors are equally contributed to this work.

${ }^{*}$ Corresponding authors: zhangjian@issp.ac.cn, lidi@issp.ac.cn, xyqin@issp.ac.cn 


\section{S-1}

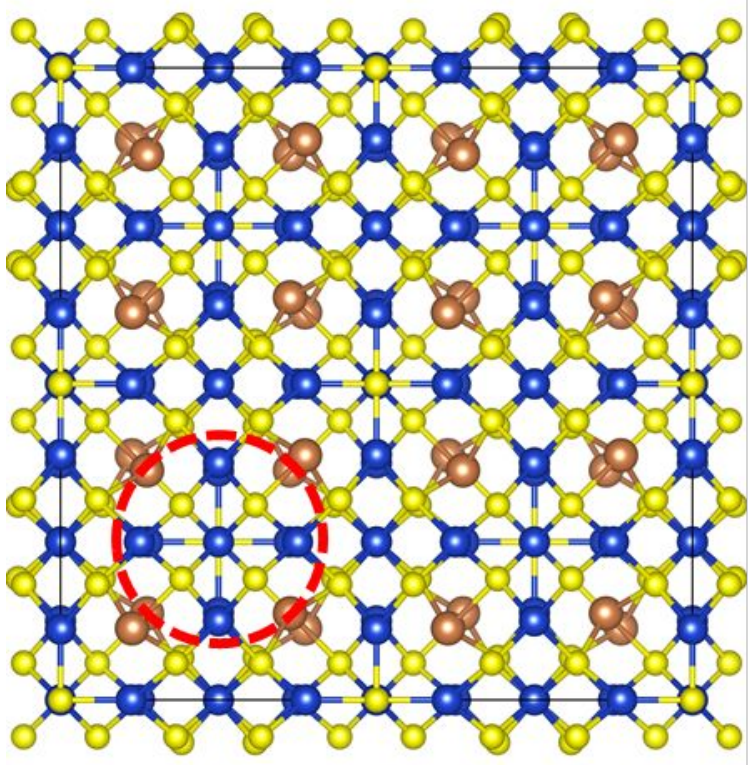

(a) $\mathrm{Cu}_{12} \mathrm{Sb}_{4} \mathrm{~S}_{13}$-supercell

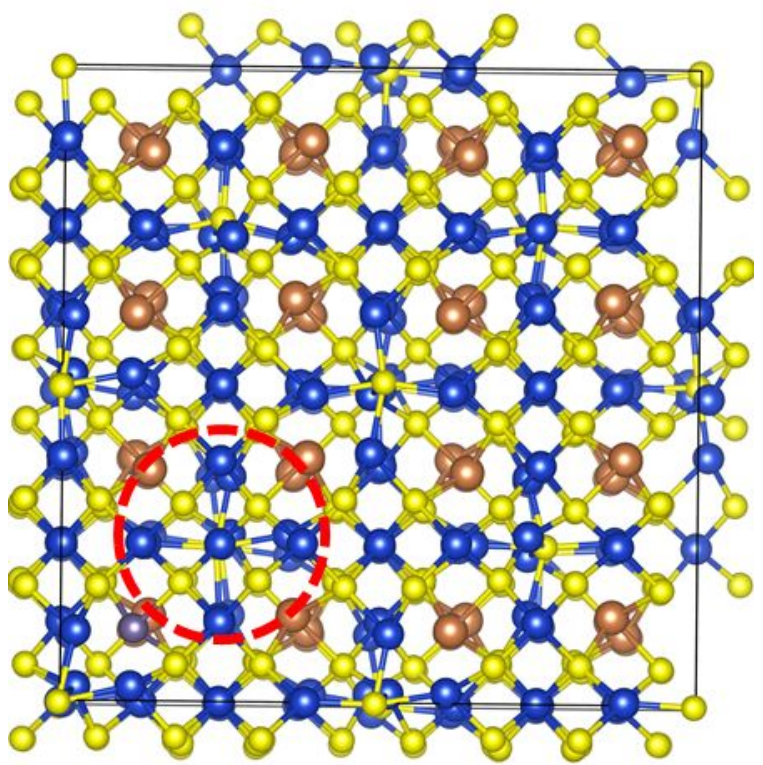

(b) $\mathrm{Cu}_{12} \mathrm{Sb}_{3.9375} \mathrm{Ge}_{0.0625} \mathrm{~S}_{13}$-supercell

Figure $\mathrm{S} 1$ The lattice structure of $\mathrm{Cu}_{12} \mathrm{Sb}_{4} \mathrm{~S}_{13}$ supercell (a) and $\mathrm{Cu}_{12} \mathrm{Sb}_{3.9375} \mathrm{Ge}_{0.0625} \mathrm{~S}_{13}$ supercell (b) crystals. Blue balls denote $\mathrm{Cu}$ ions, earth yellow denote $\mathrm{Sb}$ ions, bright yellow denote $\mathrm{S}$ ions, and silver denote Ge ions, respectively. 